\title{
Caracterización de la movilidad compartida de las empresas de redes de transporte en México: entropía, homeostasis, negentropía
}

\author{
Characterization of the shared mobility of the transport network \\ companies in Mexico: entropy, homeostasis, negentropy \\ Mario Guadalupe González Pérez ${ }^{1 *}$ \\ Recibido 27 de noviembre de 2018, aceptado 16 de agosto de 2019 \\ Received: November 27, 2018 Accepted: August 16, 2019
}

\begin{abstract}
RESUMEN
Diversos sistemas urbanos están implementando con mayor o menor éxito alternativas para la automovilidad particular, tendientes en reducir entropía en el sistema. De igual forma, estrategias implementadas in situ, por empresas de redes de transporte también han expresado homogeneidad con la negentropía de la termodinámica. Este artículo identifica isomorfismos coercitivos, normativos y miméticos de empresas de redes de transporte que operan en México, con el objetivo de determinar cualitativamente la homeostasis de la movilidad compartida en sistemas urbanos. En este sentido, se utiliza el modelo entropía-homeostasisnegentropía (EHN); una conceptualización que sub-clasifica por fases la entropía producida por el elemento antrópico, la homeostasis que presenta el sistema y la negentropía implementada para reducir la entropía. Esta instrumentación metodológica retoma el modelo presión-estado-respuesta de la OCDE y las premisas termodinámicas y fundacionales de la teoría de los sistemas. Complementariamente, son retomados los presupuestos del isomorfismo institucional, donde sus derivaciones coercitivas, normativas y miméticas en la automovilidad compartida ofertada por las empresas de redes de transporte pretenden la reducción de los costos de viaje, el uso racional del espacio, la disminución de carga vehicular, emisiones y accidentalidad en sistemas urbanos. Sin embargo, esta negentropía de efectos inmediatos deja de ser efectiva con el paso del tiempo, acorde con el segundo principio de la termodinámica. En suma, reducciones de entropía requieren implementar negentropías definitivas en las relaciones urbanono urbano y en las interacciones intra-urbanas.
\end{abstract}

Palabras clave: Empresas de redes de transporte, entropía, movilidad compartida.

\begin{abstract}
Several urban systems are implementing with greater or lesser success the alternatives for automobility, which tends to reduce the system's entropy. Similarly, the strategies implemented in situ by ridesourcing companies; have also expressed homogeneity with the negentropy of thermodynamics. This article identifies the coercive, normative and mimetic isomorphisms of transport network companies operating in Mexico, with the objective of qualitatively determining the homeostasis of shared mobility in urban systems. In this sense, the entropy-homeostasis-negentropy (EHN) model is used; a conceptualization that subclassifies in phases the entropy produced by the anthropic element, the homeostasis presented by the system and the negentropy implemented to reduce entropy. This methodological instrumentation takes up the pressure-state-response model of the OECD and the thermodynamic and fundamental premises
\end{abstract}

\footnotetext{
1 Universidad de Guadalajara, División de Ingenierías e Innovación Tecnológica. Jalisco, México. E-mail: inge_united@hotmail.com * Autor de correspondencia: inge_united@ hotmail.com
} 
of systems theory. In addition, the assumptions of the institutional isomorphism are retaken, where their coercive, normative and mimetic derivations in the shared automobility offered by ridesourcing companies aim to reduce the travel costs, the rational use of space, the reduction of the load vehicular, emissions and accidents in urban systems. However, this negentropy of immediate effects ceases to be effective with the passage of time, according to the second principle of thermodynamics. In summary, entropy reductions require the implementation of definitive negentropies in urban-non-urban relationships and intra-urban interactions.

Keywords: Transport network companies, entropy, shared mobility.

\section{INTRODUCCIÓN}

En todo sistema termodinámico, es posible identificar fuerzas entrópicas capaces de transformar muchas veces irreversiblemente las condiciones originarias de su estructura y entorno. En el ámbito urbano, la entropía ha ocurrido por permisiones u omisiones relacionadas con la planificación no sistémica y el sobreconsumo de los insumos provenientes de los sistemas no urbanos, para satisfacer los procesos de habitabilidad y movilidad. Aquí, la homeostasis en la frontera y el entorno de estos sistemas se vuelve cada vez más compleja, debido al modo de hacer la ciudad, el cual ha complicado los procesos intra e inter-sistémicos.

En materia de movilidad, diversos sistemas urbanos están implementando con mayor o menor éxito alternativas tendientes a reducir la automovilidad particular, a través de empresas que operan el denominado ridesourcing. Algunas de estas empresas de redes de transporte están incentivando el consumo compartido; es decir, negentropía termodinámica supeditada a la automovilidad. De tal forma, que este artículo identifica isomorfismos coercitivos, normativos y miméticos de servicios compartidos promovidos por estas empresas de redes de transporte, con el objetivo de determinar cualitativamente la homeostasis de la movilidad compartida en sistemas urbanos de México.

Metodológicamente, este trabajo fue estructurado en cuatro apartados: a) en primer término, se describen las relaciones termodinámicas sistema-fronteraentorno y entropía-negentropía. Posteriormente, se explica la estructura teórico-conceptual del modelo Entropía-Homeostasis-Negentropía (EHN). Este modelo representa una sub-división interesante para evaluar cualitativamente la presión, el estado y respuestas de las fuerzas entrópicas y negentrópicas.
En una tercera etapa, son revisados algunos antecedentes de la automovilidad compartida como consumo colaborativo e identificados isomorfismos coercitivos, normativos y miméticos; asimismo, se establecen condicionantes de entropía, homeostasis y negentropía de la movilidad compartida en sistemas urbanos de México. Finalmente, se presentan conclusiones asociadas con la negentropía de corto plazo; es decir, alternativas de efectos esporádicos, ocasionales o inmediatos. Aquí, estas negentropías dejan de ser efectivas con el paso del tiempo y se vuelven más temprano que tarde, homogéneas al segundo principio de la termodinámica. En este sentido, la reducción de entropía en materia de movilidad urbana conlleva la implementación de negentropías definitivas en las relaciones urbano-no urbano y en las interacciones intra e inter-urbanas, asociadas con la des-incentivación de la automovilidad. En suma, la automovilidad compartida no resuelve el problema de fondo y actúa como negentropía ocasional dentro del marco conceptual del modelo EHN.

\section{ENTROPÍA Y NEGENTROPÍA: EL URBANO, PERIURBANO Y NO URBANO}

Las réflexions sur la puissance motrice du feu et sur les machines propres à déveloper cette puissance [1] de Sadi Carnot en 1824 y la introducción en 1865 del concepto entropía por Rudolf Clausius, formalizan el segundo principio de la termodinámica de los procesos irreversibles. Posteriormente, los aportes de Ludwig Boltzmann en mecánica estadística facilitan la comprensión del término, a través de la ecuación $\mathrm{S}=\mathrm{K} \log \mathrm{W}$; donde $S$ simboliza el concepto termodinámico de entropía, $K$ representa la constante de Boltzmann que relaciona la concepción de la mecánica estadística y la termodinámica clásica y $W$ el número de micro-estados posibles de ocurrencia. Sobre esta ecuación, Schrödinger puntualizaba: "Let 
me recall that entropy is a direct measure of molecular disorder, viz. its logarithm" [2]. En esta fórmula, Varadhan afirma que la entropía (S) se entiende "como el logaritmo del número de microestados que corresponde a un estado macro" [3].

A mediados del siglo XX, la definición de entropía utilizada por décadas en el estudio de sistemas cerrados y aislados, es conceptualizada ahora desde las nociones de la teoría de la comunicación de Shannon y Weaver [4]. Para Shannon, la entropía representa "(...) una medida de la información o incertidumbre de experimentos probabilísticos arbitrarios" [5], que puede ser utilizada como entropía negativa y reducir con ello, niveles de entropía en el sistema [6]. De esta manera, sí la información o entropía negativa está asociada con una medida del orden [7] definida "por una expresión isomorfa con la entropia negativa de la termodinámica” [8]; entonces, “(...) la falta de información corresponde a un desorden real en los grados de libertad buscados" [9]. No obstante, para Kish y Ferry “(...) there is no general interrelation between the information entropy and the thermodynamical entropy (...) the information entropy is a subjective, measurement-system-dependent feature while the thermodynamical entropy is an objective property of the physical system" [10].

En este contexto, todo sistema cerrado o aislado constituido por materia inanimada produce entropía en función del tiempo. En estos sistemas, la entropía incrementa el desorden y disminuye el orden intra-sistémicos, debido a una ausencia de intercambios de materia y energía en sistemas aislados y restricciones en el intercambio de materia más no de energía en sistemas cerrados. Por ello, la materia inanimada tiende hacia este estado de entropía creciente y orden decreciente [8]. Sin embargo, esta tendencia puede revertirse bajo ciertas circunstancias en los denominados sistemas abiertos y en algunos sistemas semi-abiertos o semi-cerrados como los sistemas urbanos. Los sistemas urbanos se comportan semi-abiertamente o semi-cerradamente y presentan complejas singularidades asociadas por ejemplo, con el crecimiento urbano-habitacional o la automovilidad. El crecimiento urbano-habitacional no puede prolongarse infinitamente, tanto horizontal como vertical, a causa de la existencia de límites geográficos y/o restricciones del entorno. Igualmente, un aumento ilimitado de la automovilidad colapsaría físicamente las arterias del subsistema vial y comprometería drásticamente las variables velocidad y tiempo de la conocida ecuación física: $v=d / t$.

Por otro lado, los sistemas abiertos y semi-abiertos o semi-cerrados no sólo producen entropía, sino también la importan. Esta importación puede ser negativa (negentropía), debido a propiedades intrínsecas para subsanar desequilibrios energéticos. Los sistemas vivos por ejemplo, son una variación de sistemas abiertos capaces de alimentarse del orden existente en el entorno, y lograr vencer, al menos temporalmente el segundo principio de la termodinámica [11]. En resumen, relaciones intrasistémicas e interacciones sistema-entorno suponen el nacimiento de posibles emergencias; es decir, de estructuras capaces de mantener el sistema en el tiempo, por medio de la disipación de los excedentes energéticos [12].

Los sistemas vivos, son sistemas abiertos que contienen estructuras disipativas, y "están en condiciones de dar sorpresas, asumiendo conductas macroscópicas inesperadas, estructurándose en formas espaciales no previstas" [13]. Concretamente, "en estos tipos de estructuras y orden se basan la vida, la síntesis de proteínas, la generación del impulso nervioso o la contracción muscular, la organización de un termitero, los ecosistemas y las propias organizaciones humanas" [14]. Por ello, la denominada Teoría de los Sistemas no solo ha pretendido responder a la ontología de los sistemas, sino paulatinamente ha configurado una epistemología sistémica, o teoría del conocimiento de los sistemas, que sirve también como método para la comprensión de la estructura, funcionamiento, complejidad y relaciones de los sistemas. En sus propios términos, es un sistema teórico constituido por subsistemas conceptuales, formados a su vez por otros sistemas diferenciados de su entorno inmediato.

En el urbanismo, el enfoque de sistemas no ha estado ausente. Desde hace años, se han abordado desde los presupuestos de la Teoría General de los Sistemas (TGS), diversas investigaciones que tratan anomalias asociadas con el hábitat residencial urbano [15], que han llevado al desarrollo de indicadores para que las ciudades entendidas como sistemas aspiren a convertirse en un modelo de ciudad sostenible [16]. En este sentido, el desarrollo es visto como una emergencia sistémica [17], y la 
planeación urbana pasa a ser concebida desde lo sistémico y complejo. Sin embargo, no siempre ha resultado sencillo determinar con precisión in situ el funcionamiento, ubicación y límites de los elementos y del propio sistema urbano.

Efectivamente, el pensamiento sistémico ha evolucionado a lo largo del siglo XX en el planeamiento y práctica urbanas [18-20], y ha cuestionado el ejercicio actual de la arquitectura [21]. En este entendido, recién iniciado el siglo XXI aparecen importantes estudios sobre la complejidad de los sistemas urbanos [22], la entropía generada por la construcción de la ciudad y sus respectivas manifestaciones de orden y desorden; hasta llegar a concebir la ciudad como un sistema complejo alejado del equilibrio [23], “(...) que degrada energía porque de dicha degradación depende el orden, es decir, el funcionamiento y la estructura de la misma" [24]. De ahí, se vuelve plausible la identificación de tres conceptos clave e interdependientes entre sí: el sistema, la frontera y el ambiente; los cuales pueden ser esquematizados y re-conceptualizados convenientemente desde lo urbano, tal y como se muestra en la Figura 1.

En la figura anterior, el sistema representa la ciudad o el urbano, los sistemas de soporte representan el ambiente, entorno o sistema(s) no urbano(s) y la frontera o límites, el periurbano. El urbano está constituido por materia, energía e información, homogéneo al resto de sistemas que

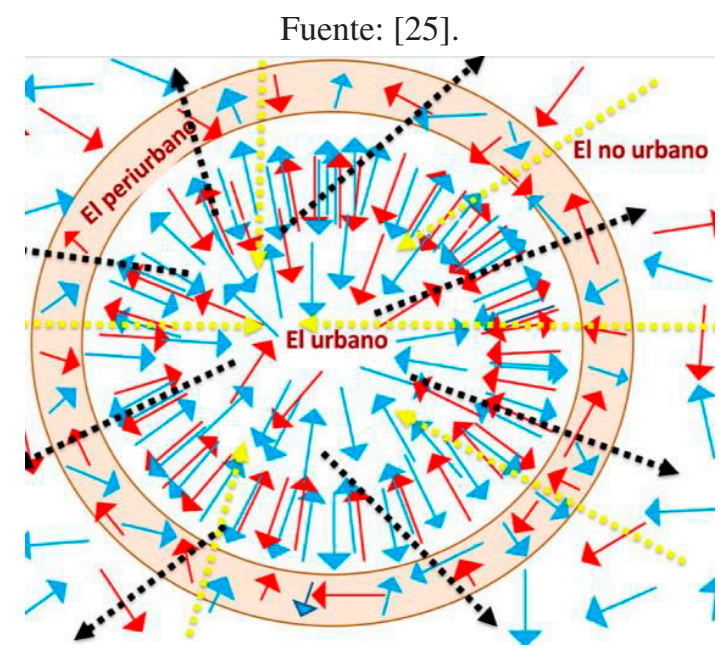

Figura 1. Fuerzas entrópicas y negentrópicas en el urbano, periurbano y no urbano. constituyen el mundo. Este mundo circunscribe al conjunto de fenómenos observables que exhiben una uniformidad estructural y se manifiestan por muestras de orden isomorfas en sus diferentes niveles o reinos [8]. Dentro de las cualidades del urbano, una peculiar es producir la urbanización. Esta actividad implica transformar y consumir sistemas no urbanos ricos en materia y energía (negentropía) indispensable para su sobrevivencia y funcionamiento. Sin embargo, este proceso traslada parte de la entropía producida al no urbano. En suma, el urbano produce desorden y hasta ciertos límites responde con orden; además, tiene límites establecidos por el espacio y el tiempo; es decir, mientras el urbano no alcance tales límites, su frontera probablemente estará desplazándose hacia el no urbano; difuminando la divisoria urbanono urbano, e hibridando y configurando nuevas complejidades sistémicas.

En la divisoria entre el urbano y el no urbano ocurre la periurbanización. Su constitución física se asemeja a una franja con cualidades urbanas, no urbanas e híbridas. Esta franja también conocida como el periurbano concentra singularidades no encontradas en el urbano y en el no urbano. En este sentido, el periurbano es un segmento curvo, donde se da la transición de unas condiciones iniciales a unas modificadas. No obstante, una vez pasando este segmento se encuentra el no urbano; es decir, el espacio geográfico conformado por subsistemas no modificados visualmente por la actividad antrópica. Aquí, encontramos la materia biótica y abiótica; la cual tiene sus propios mecanismos naturales de regulación (resiliencia) y conservación. Sus niveles de entropía han logrado mantenerse por millones de años, debido a la evolución y complejidad de sus propiedades naturales para generar negentropía.

\section{DEL MODELO PER AL MODELO EHN}

A finales de la década de los setentas, David Rapport y Anthony Friend cimentan las bases del modelo presión-estado-respuesta (PER) en el trabajo Towards a comprehensive framework for environmental statistics: a stress-response approach [26]. Este esquema causa-efecto considera los sistemas de soporte como proveedores de insumos para el funcionamiento del sistema de presión y receptores de una parte significativa de la materia y energía no utilizadas para producir trabajo (entropía). No 
obstante, en esta abstracción la fuerza antrópica también es capaz de dar respuesta a la entropía producida y minimizar grados de intensidad de los efectos generados. "Estas respuestas a su vez actúan sobre las presiones, dando lugar a un proceso continuo, que se conoce como proceso de reevaluación" [27]. En suma, el PER cuestiona: ¿qué está ocurriendo con el ambiente?, ¿por qué está ocurriendo? y ¿qué se está haciendo al respecto? [28].

El modelo PER es incorporado por la Organización para la Cooperación y Desarrollo Económico (OCDE), con el objetivo de contribuir con la armonización de las iniciativas ambientales en sus países miembros, mediante el desarrollo de una serie de parámetros o indicadores ambientales, circunscritos en un marco conceptual basado en una comprensión común de conceptos y definiciones [29]. "Estes indicadores estão clasificados segundo o modelo PER: i) indicadores das pressões, diretas e indiretas, sobre o meio ambiente; ii) indicadores das condições ambientais, e iii) indicadores das respostas da sociedade" [30]. Con esta base, el modelo entropía-homeostasis-negentropía (EHN) está fundamentado en la TGS y en la lógica del modelo PER. El modelo EHN considera que el urbano contiene fuerzas que causalmente incrementan su entropía e introducen entropía en otros subsistemas ubicados en el no urbano. De tal forma, que subdivide la entropía en tres fases: i) entropía subcrítica, ii) entropía crítica y ii) entropía hipercrítica.

En la fase subcrítica, los niveles de entropía no inhiben el funcionamiento y operatividad del sistema urbano y sus elementos. El sistema urbano trabaja a pesar de las anomalías presentes. Aquí, los mecanismos de auto-regulación no se encuentran severamente afectados; por tanto, el sistema es capaz de revertir la entropía auto-producida o inducida por agentes externos, a través de sus propias estructuras de disipación. De esta manera, mediante la introducción de negentropía proveniente del no urbano, o de entornos inmediatos a sus subsistemas, es posible el desarrollo de emergencias capaces de adaptarse a las nuevas condiciones. Sin embargo, cuando los niveles de entropía se encuentran en fase crítica se presentan disfunciones intra-sistémicas, donde algunos elementos no logran recuperase, mientras otros en cambio, logran hibridar y adaptarse a las nuevas condiciones. Finalmente, en fase hipercrítica se han afectado irreversiblemente la estructura y elementos del urbano; incluso, la afectación puede sobrepasar el periurbano hasta afectar elementos del no urbano [25].

La homeostasis por su parte, es un término popularizado por Walter Canon a finales de los años veinte y se relaciona con el estado interior del sistema. Este concepto hace referencia a un sistema vivo ante la interacción con su entorno. Es decir, "Changes in the surroundings excite reactions in this system, or affect it directly, so that internal disturbances of the system are produced" [31]. En este sentido, el sistema urbano presenta tres fases de homeostasis: i) homeostasis en fase reversible, ii) homeostasis en fase cuasi-reversible y iii) homeostasis en fase irreversible. La fase de homeostasis reversible tiene la propiedad de revertir afectaciones en sus elementos y/o relaciones, a través de la disminución de niveles de entropía; en tanto, la homeostasis en fase cuasi-reversible contiene daños parciales en algunos elementos y/o relaciones del sistema. Aquí, no es posible revertir todas las afectaciones. Finalmente, la homeostasis en fase irreversible es característica de sistemas termodinámicamente cerrados o aislados; es decir, esta condición imposibilita al urbano revertir las afectaciones en sus elementos y/o relaciones [25].

Por otro lado, desde la década de los cuarenta del siglo pasado, el físico-teórico Erwin Schrödinger exponía que los sistemas vivos necesitaban alimentarse de entropía negativa para reducir los niveles de entropía [2]. Años más tarde, este concepto era identificado por el término negentropía. No obstante, sobre este término se han propuesto dos principios: a) Principio de Negentropía para la existencia del orden dinámico y b) Principio de Máxima Producción de Negentropía (PMNEP) para la evolución del orden dinámico. En otros términos, "These two additional principles now enable unified explanation of order creation, existence, evolution, and destruction; using thermodynamics" [32].

En el modelo EHN es posible minimizar entropía a través de negentropía y mantener la homeostasis en estado estable. Esto debido a que "living systems, maintaining themselves in a steady state, can avoid the increase of entropy, and may even develop towards states of increased order and organization" [8]. De esta forma, la teoría del modelo EHN presenta tres fases de negentropía: i) 
negentropía inexistente, ii) negentropía ocasional y iii) negentropía definitiva. La fase identificada por una inexistencia de negentropía aumenta el desorden causado por el tiempo y operación de los elementos intra-sistémicos. Aquí, el urbano no responde al desgaste inminente de las operaciones sistémicas, y presenta por tanto, anomalías que van incrementándose hasta el cese de sus procesos.

En el caso de la fase ocasional, el urbano logra disminuir temporalmente los niveles de entropía auto-producida; sin embargo, sus efectos positivos son esporádicos y de corto plazo. Esta fase suele ser muy común en sistemas urbanos con isomorfismos asociados con la automovilidad particular, donde el origen del problema radica en que las intervenciones en el espacio público no atienden las causas, sino solo los efectos [25]. De ahí que, se vuelve necesario implementar negentropía en fase definitiva, para minimizar los niveles de entropía y mantener la homeostasis del sistema en condiciones óptimas. En suma, el EHN proporciona una sub-clasificación que posibilita la medición cualitativa de la estructura del urbano conformada por entropía, homeostasis y negentropía, tal y como se muestra en la Figura 2.

\section{TECNOLOGÍAS DE LA INFORMACIÓN Y COMUNICACIÓN, ECONOMÍA COLABORATIVA Y AUTOMOVILIDAD COMPARTIDA}

Resulta sencillo constatar la relevancia que las denominadas tecnologías de la comunicación e información (TICS) representan en la vida cotidiana

Fuente: [25].
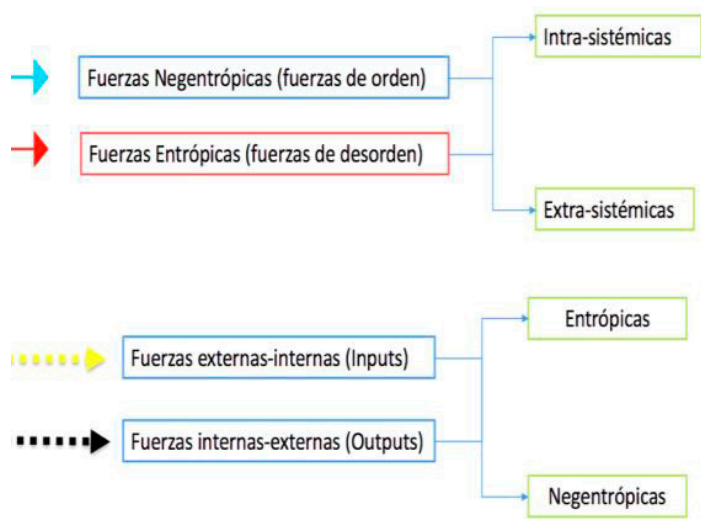

Figura 2. Subclasificaciones del modelo EHN. de las personas que habitan el espacio geográfico de diversos sistemas urbanos. En estos sistemas, la telefonía móvil, el internet y las redes sociales virtuales han configurado nuevas formas de intercomunicación hablada y/o escrita. Los teléfonos inteligentes por ejemplo, han posibilitado junto al internet el acceso al espacio virtual o ciberespacio; "un lugar no físico donde están ocurriendo infinidad de situaciones de la vida cotidiana" [33]. Es por ello, que resulta plausible afirmar que actualmente la sociedad “(...) está inmersa en cambios cotidianos impuestos por las tecnologías de la comunicación y la información (....)” [34].

Este boom tecnológico-comercial empezó a tomar auge a mediados de los años noventa del siglo pasado. Por ese tiempo, diversos gobiernos y empresas a nivel global se percatan de las ventajas proporcionadas por el internet y el nuevo paradigma de organización económica; toda vez, que se vuelve innecesario contar con bases permanentes o temporales de trabajadores y proveedores, debido a que este modelo prioriza las redes de sujetos y organizaciones y le resta preponderancia al sujeto en lo individual [35]. No obstante, no es sino a partir de What's Mine is Yours: The Rise of Collaborative Consumption [36] y The Zero Marginal Cost Society: the Internet of Things, the Collaborative Commons and the Eclipse of Capitalism [37], que se establecen los principios de una nueva disciplina que exhortaba otra forma de hacer economía.

Efectivamente, décadas previas a la formalización de esta disciplina, surgieron programas de consumo colaborativo; por ejemplo, en materia de movilidad compartida. En este campo, desde finales de los años cuarenta aparecieron indicios del denominado carsharing, y entre los sesentas y setentas del siglo pasado, el bikesharing, a través de programas como Witte Fietsenplan, Witkarren, StattAuto [38-39]. Desde luego, por esos años se carecía del potencial del internet, las redes sociales virtuales y las plataformas tecnológicas; de tal forma, que no tuvieron el auge esperado. Sin embargo, en la actualidad, "estamos siendo copartícipes de nuevos modos de convivencia que no excluyen los temas relacionados con la movilidad y los sistemas de transporte urbano" [33]. Estas transformaciones de la tecnología en el sector transporte han llevado actualmente a tener "The ability to conveniently request, track, and pay for trips via mobile devices is changing the way people get around and interact with cities" [40]. 
En materia de movilidad, disciplinas como la sociología, psicología, geografía y sociedad de la información han aportado enormes series de datos a la configuración de la denominada geografía de la movilidad; una derivación surgida con base en los supuestos de la geografía del transporte que pretendían favorecer la planificación y ordenamiento territorial [41]. No obstante, en diversos sistemas urbanos con características isomorfas asociadas con la automovilidad particular han experimentado mayores tasas de motorización y han consolidado cadenas de producción dependientes del consumo del vehículo particular, con saldos a favor de unos y detrimento en la calidad de vida de otros. De tal manera, que el tema de la automovilidad particular preocupa al grado que se vuelve impostergable formular alternativas menos entrópicas como las propuestas por la denominada movilidad compartida. Según Cohen y Shaheen, la movilidad compartida incide en distintas facetas de la planificación urbana: a) transporte y circulación, b) zonificación, uso de la tierra y gestión del crecimiento, c) diseño urbano, d) vivienda, e) desarrollo económico y f) política ambiental, conservación y acción climática [42].

En relación con la automovilidad compartida, destacan comercialmente empresas como Uber, la cual ha promovido el servicio uberpool. Lyft, a través de su servicios Driver Destination service y Lyft Carpool, BlaBlaCar para trayectos de larga distancia, el servicio Didi Bus de la fusionada Didi Chuxing, algunas modalidades de Grab como GrabShare o Grabcar de 6 plazas, Sidecar, Car2go, eGo, CarShare, el Ola Share de Ola Cabs, ReachNow entre otras empresas que ofrecen de momento, solo la subcontratación del servicio de transporte tradicional (Cabify, Taxibeat, Easy taxy, City Drive, otros).

Estos servicios de automovilidad compartida contienen isomorfismos origen-trayecto-destino en sus viajes; toda vez que comparten de diversas formas el servicio y/o la unidad a un costo menor que el normal; es decir, que el servicio no compartido. Además, la fundamentación de estas alternativas de automovilidad compartida tienen como común denominador la optimización del uso del espacio público, al circular un menor número de unidades vehiculares propias o alquiladas. Igualmente, reducir tráfico, emisiones contaminantes, ruido, combustible, tiempo, espacios de estacionamiento y número de accidentes [43]. Sobre la probabilidad de accidentes, es pertinente suponer que “(...) entre más elementos estén en el juego, más se incrementa la posibilidad de un suceso no necesariamente aleatorio" [34].

En el servicio peer-to-peer carsharing, los propietarios de vehículos alquilan temporalmente sus automóviles a personas ubicadas en su área circundante [44]; es decir, mediante el carsharing se proporciona a los usuarios automóviles de una flotilla según las necesidades [45]. En tanto, el carpooling, “(...) is a scheme according to which several people share a common vehicle simultaneously, in order to reach common, or nearby destinations" [43]. En este sentido, varias de estas modalidades se combinan y/o complementan. Por ejemplo, un usuario puede solicitar un servicio de ridesourcing en su modalidad de carpooling; disminuyendo con ello, el costo del viaje.

En el caso de México, existen otras modalidades de automovilidad compartida que de alguna manera han modificado el ejercicio de la movilidad urbana motorizada; tales como, acuerdos verbales informales o formales entre usuarios, o mediante la utilización de plataformas de acceso controlado, que pretenden compartir el vehículo particular para reducir los costos del viaje. Estas modalidades de movilidad motorizada compartida es posible encontrarlas en comunidades, donde sus integrantes se turnan el uso del vehículo particular por día/semana/mes. El ejercicio es realizado sin fines de lucro, donde el conductor asume los costos del desplazamiento; o bien, recibe aportaciones voluntarias y/o cuotas proporcionales al costo del viaje. Sin embargo, en ciertas ocasiones, la práctica de esta actividad es realizada por el simple hecho de compartir el vehículo. En estos casos, el propietario del vehículo decide compartir en el origen o en algún punto del trayecto el viaje, con uno o más usuarios que tienen el mismo destino. Este ejercicio es comúnmente observado en círculos sociales donde sus integrantes se conocen, o al menos tienen datos suficientes que les permitan un nivel de confianza mínimo para compartir su vehículo.

\section{ISOMORFISMOS COERCITIVOS, MIMÉTICOS Y NORMATIVOS DE LA AUTOMOVILIDAD COMPARTIDA EN MÉXICO}

Diversos sistemas urbanos en México están presentando cambios isomorfos asociados con 
la automovilidad compartida. Estos pueden ser agrupados por conveniencia en tres categorías: a) isomorfismos coercitivos, b) isomorfismos normativos y c) isomorfismos miméticos. Efectivamente, estas presunciones han sido retomadas del llamado isomorfismo institucional de DiMaggio y Powell, quienes han teorizado estos conceptos en el campo de las organizaciones. Según estos autores, es posible identificar un isomorfismo coercitivo que deriva de la influencia política y el problema de la legitimidad; un isomorfismo mimético resultante de respuestas estándar a la incertidumbre y un isomorfismo normativo asociado a la profesionalización [46].

En este contexto, considerando estos presupuestos, se ha planteado que las organizaciones son sometidas a presiones institucionales al grado de generar homogeneidad. De ahí, la búsqueda de legitimidad y/o apoyo social para obtener los recursos necesarios para su sobrevivencia [47]. El término legitimidad circunscribe "una concepción generalizada o asunción de que las acciones de una entidad son deseables, convenientes o apropiadas dentro de un sistema socialmente construido de normas, valores, creencias y definiciones" [48]. De tal forma, que en términos sistémicos estas organizaciones o subsistemas presionados no solo por entropía intrasistémica sino por entropía intersistémica requieren legitimidad (negentropía) para mantenerse en el tiempo. En este sentido, Bertalanffy plantea que el isomorfismo está relacionado con similaridades estructurales, consecuencia de la existencia de propiedades generales en todo sistema [8]. Por ello, toda tendencia al isomorfismo empuja hacia la homogeneidad [49]; es decir, "The concept that best captures the process of homogenization is isomorphims" [46].

El isomorfismo coercitivo circunscribe fuerzas entrópicas provenientes del no urbano y de sus propios elementos; las cuales, direccionan la réplica de comportamientos y funcionamientos, para mantener en el espacio-tiempo la homeostasis reversible de las relaciones urbano-no urbano y de las propias relaciones intra-urbanas. El isomorfismo mimético por otro lado, considera la incertidumbre como causa de entropía intrasistémica. De tal forma que ante el desconocimiento del no urbano, estos subsistemas se ven en la necesidad de implementar instrumentos y/o mecanismos realizados por otros elementos del urbano como una especie de imitación del éxito; es decir, una medida negentrópica necesaria para reducir los niveles de entropía auto-producida y poder mantener continuidad a lo largo del tiempo. $\mathrm{El}$ isomorfismo normativo por su parte, se legitima con base en la profesionalización de los elementos del sistema. Este isomorfismo, “(...), involucra la mimetización de normas establecidas" [50], donde el urbano implementa negentropía para mantener su homeostasis. Aquí, el urbano, a través de modelos pretende controlar los excedentes energéticos (entropía) y sostener con cierta seguridad el funcionamiento óptimo de sus procesos habituales.

Metodológicamente este trabajo identificó documentalmente las principales expresiones de automovilidad compartida: peer-to-peer, carsharing, carpooling y ridesourcing. Posteriormente, elegida por conveniencia la actividad del ridesourcing; cuya introducción en el mercado de subcontratación de este servicio en diversos sistemas urbanos de México (Ciudad de México, Guadalajara, Monterrey, otras), ha ocasionado singularidades e isomorfismos en años recientes, abstrae homogeneidades de tipo coercitivo, mimético y normativo, para realizar un análisis estructural y relacional desde la lógica del modelo EHN. Finalmente, fueron determinadas cualitativamente las fases de entropía, homeostasis y negentropía de la automovilidad compartida en las tres principales metrópolis del país, tal y como se muestra en la Figura 3.

Con base en el esquema de la Figura 3, se indican en las Tablas 1 y 2 , los isomorfismos, entropías, homeostasis y negentropías asociadas con la automovilidad compartida en la Ciudad de México, Guadalajara y Monterrey.

\section{CONCLUSIONES}

Este trabajo ha asumido que al interior del sistema urbano ocurren relaciones entre sus subsistemas y sus respectivos ambientes. Aquí, el proceso de interacción implica consumo de materia y energía; por tanto, producción de entropía. No obstante, la forma de hacer ciudad, vivir la ciudad o moverse en la ciudad puede acelerar o desacelerar la entropía de los procesos y elementos intra-sistémicos y/o afectar elementos y relaciones de sistemas no urbanos. En resumen, la entropía repercute en la homeostasis del sistema urbano y de los sistemas no urbanos. 
Fuente: Elaboración propia.
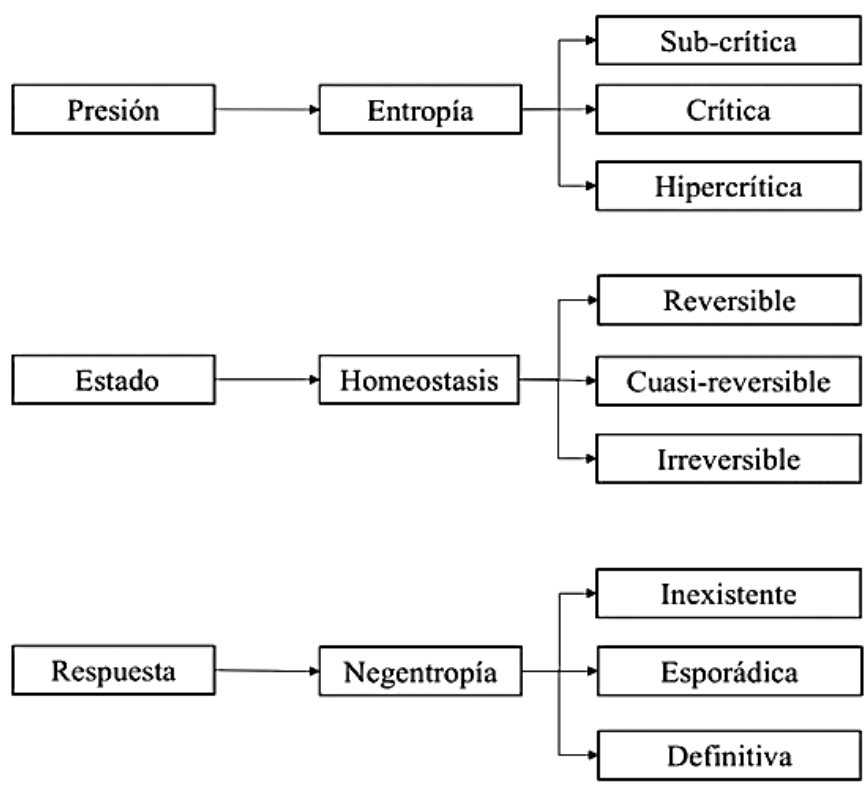

Figura 3. EHN y automovilidad compartida las principales metrópolis de México.

Tabla 1. Isomorfismos de empresas de redes de transporte.

\begin{tabular}{|l|l|l|}
\hline \multicolumn{1}{|c|}{ Coercitivos } & \multicolumn{1}{|c|}{ Miméticos } & \multicolumn{1}{c|}{ Normativos } \\
\hline $\begin{array}{l}\text { Imposiciones jurídicas en materia de } \\
\text { regularización de la aplicación tec- } \\
\text { nológica, a través de direccionamientos } \\
\text { sobre el acceso, uso y pago. }\end{array}$ & $\begin{array}{l}\text { Reacciones en beneficio del usuario de } \\
\text { estas empresas ante posicionamientos de } \\
\text { rechazo por los prestadores tradicionales } \\
\text { del servicio de transporte. }\end{array}$ & $\begin{array}{l}\text { Operación sustentada en la } \\
\text { base social del usuario y la } \\
\text { lucha de los proveedores } \\
\text { para definir las condiciones } \\
\text { de su trabajo. }\end{array}$ \\
\hline $\begin{array}{l}\text { Sanciones por incumplimiento en ma- } \\
\text { teria de registro de los proveedores del } \\
\text { servicio de transporte a través del uso } \\
\text { de aplicaciones tecnológicas. }\end{array}$ & $\begin{array}{l}\text { Adecuaciones del servicio para asegurar } \\
\text { la permanencia y preferencia ante la } \\
\text { competencia (flexibilización en el méto- } \\
\text { do de pago). }\end{array}$ & $\begin{array}{l}\text { Legitimación del servicio en el } \\
\text { imaginario social del usuario, } \\
\text { basado en la configuración } \\
\text { de niveles de confianza } \\
\text { aplicación-conductor. }\end{array}$ \\
\hline $\begin{array}{l}\text { Prohibición en la operación de estos } \\
\text { servicios en sistemas urbanos propensos } \\
\text { a la presión e intereses de los servicios } \\
\text { tradicionales de transporte. }\end{array}$ & $\begin{array}{l}\text { Homogeneidad en la calidad del servicio } \\
\text { de transporte ofertado } \\
\text { taxificación del ridesourcing). }\end{array}$ & $\begin{array}{l}\text { Institucionalización del servicio } \\
\text { a través de la regulación formal } \\
\text { de su operación por parte de la } \\
\text { autoridad. }\end{array}$ \\
\hline $\begin{array}{l}\text { Configuración de servicios clandestinos, } \\
\text { al margen de los intermediarios tecno- } \\
\text { lógicos y el conocimiento u omisión por } \\
\text { parte de la autoridad. }\end{array}$ & $\begin{array}{l}\text { Penetración y conformación de nuevas } \\
\text { empresas de redes de transporte con } \\
\text { esquemas mayormente flexibles; pero } \\
\text { con incertidumbre en el éxito de las } \\
\text { empresas que previamente han acaparado } \\
\text { el mercado. }\end{array}$ & \\
\hline
\end{tabular}

Fuente: Elaboración propia.

En el caso de México, la movilidad es una necesidad indispensable pero genera entropía. De tal forma, que responder a esta entropía en las principales metrópolis ha generado una variedad de alternativas negentrópicas supeditadas algunas al paradigma del automóvil; otras sin embargo, se deslindan 
Tabla 2. Entropía Homeostasis y Negentropía de la automovilidad compartida.

\begin{tabular}{|l|l|l|}
\hline \multicolumn{1}{|c|}{ Entropía } & \multicolumn{1}{|c|}{ Homeostasis } & \multicolumn{1}{c|}{ Negentropía } \\
\hline $\begin{array}{l}\text { Permisión u omisión en la ope- } \\
\text { ración formal e informal del } \\
\text { ridesourcing. }\end{array}$ & $\begin{array}{l}\text { Complicaciones viales cuasi- } \\
\text { reversibles en horas valle y } \\
\text { colapsos en horas pico en el } \\
\text { ejercicio de la automovilidad } \\
\text { compartida. }\end{array}$ & $\begin{array}{l}\text { Reducción de la automovilidad a través } \\
\text { de subsistemas de transporte colectivo } \\
\text { sustentables e implementación de programas } \\
\text { de movilidad urbana sostenible. }\end{array}$ \\
\hline $\begin{array}{l}\text { Presunción de negentropía en el } \\
\text { consumo de la automovilidad } \\
\text { compartida. }\end{array}$ & Beneficios de corto plazo. & $\begin{array}{l}\text { Implementación de negentropías definitivas, } \\
\text { por medio de subsistemas de transporte } \\
\text { colectivo no motorizados. }\end{array}$ \\
\hline $\begin{array}{l}\text { Inducción al consumo de ele- } \\
\text { mentos necesarios para la opera- } \\
\text { tividad y funcionamiento de los } \\
\text { subsistemas de autotransporte } \\
\text { compartido (vehículo). }\end{array}$ & $\begin{array}{l}\text { Interdependencia en la cadena } \\
\text { de producción y consumo. }\end{array}$ & $\begin{array}{l}\text { Reingeniería en las cadenas de suministro, } \\
\text { a través de incentivos para subsistemas no } \\
\text { motorizados y políticas públicas en materia } \\
\text { de movilidad no motorizada. }\end{array}$ \\
\hline $\begin{array}{l}\text { Desgaste de la infraestructura } \\
\text { vial (camino). }\end{array}$ & $\begin{array}{l}\text { Superficies de rodamiento defi- } \\
\text { cientes y riesgosas. }\end{array}$ & $\begin{array}{l}\text { Programas de inversión de infraestructura } \\
\text { para la movilidad peatonal y ciclista. } \\
\text { Reducción en inversión en infraestructuras } \\
\text { para la automovilidad. }\end{array}$ \\
\hline
\end{tabular}

Fuente: Elaboración propia.

de su uso. Dentro de las primeras han aparecido empresas de redes de transporte que ofrecen diversas modalidades en sus servicios; Uber por ejemplo, ofrece la realización de desplazamientos compartidos, donde es posible identificar isomorfismos coercitivos, normativos y miméticos relacionados con este ejercicio; toda vez, que los servicio de automovilidad compartida pretenden la reducción de los costos de viaje, el uso racional del espacio, la disminución de carga vehicular en las vías, reducción de emisiones contaminantes y disminuciones en la accidentalidad con base en la probabilidad. Sin embargo, está demostrado que esta negentropía deja de ser efectiva con el paso del tiempo, acorde con el segundo principio de la termodinámica, debido a que su permisión, omisión, promoción o presunción inducen al consumo de elementos necesarios para la operatividad y funcionamiento de los subsistemas de autotransporte compartido. Esta práctica no está exenta al desgaste, tanto en los vehículos como en el camino; actúa además, para resolver problemas de corto plazo. Es en suma, una negentropía ocasional conforme al modelo EHN.

En función de lo anterior, la reducción de entropía implica la implementación de negentropías definitivas en las relaciones urbano-no urbano y en las interacciones intra-urbanas de estas ciudades metropolitanas y de diversos sistemas urbanos con características isomorfas. De tal forma, que la principal contribución de este trabajo radica en la posibilidad de incorporar un nuevo marco conceptual al estudio de la movilidad urbana; es decir, considerar la estructura del modelo EHN para comprender el comportamiento termodinámico de los sistemas urbanos. Efectivamente, no siempre es posible determinar con precisión la magnitud de los efectos o el resultado de las intervenciones en alguna porción del sistema; no obstante, es posible sí al menos impulsar la inclusión, favorecer la resiliencia del sistema, incorporar premisas de sustentabilidad al diseño y configurar directrices de una planificación sistémica de la ciudad. La planificación sistémica considera los presupuestos termodinámicos de los sistemas y hace hincapié en la entropía como característica natural de los sistemas.

\section{REFERENCIAS}

[1] S. Carnot. "Réflexions sur la puissance motrice du feu et sur les machines propres à développer cette puissance". Annales scientifiques de l'École Normale Supérieure. Série 2, Tome 1, pp. 393-457. 1872.

[2] E. Schrödinger. “¿What is life?”. Cambridge University Press. Cambridge, E.E.U.U. 1944.

[3] R. Varadhan. "Entropy and many avatars". J. Math. Soc. Japón. Vol. 67, Issue. 4, 
pp. 1845-1857. May, 2015. DOI: 10.2969/ jmsj/06741845.

[4] C. Shannon and W. Weaver. "The mathematical theory of communication". University of Illinois Press. Urbana, E.E.U.U. 1949.

[5] L. Pardo. "Teoría de la información estadística”. Estadística Española. Vol. 35 No 133, pp. 195-268. 1993.

[6] A. Marcos. "Información y entropía". Arbor: Ciencia, pensamiento y cultura. Vol. $1 \mathrm{~N}^{\circ} 549$, pp. 111-138. 1991.

[7] N. Wiener. "Cybernetics: or Control and Communication in the Animal and Machine". Mass: MIT. Press. Cambridge, E.E.U.U. 1948.

[8] L. Bertalanffy. "General system theory. Foundations, Development, Applications". George Braziller. New York, E.E.U.U. 1968.

[9] L. Brillouin. "Science and information theory". Academic Press. London, England. 1962.

[10] L. Kish and D. Ferry. "Information entropy and thermal entropy: apples and oranges". Journal of Computational Electronics. Vol. 1, Issue 8, pp. 1-8. July, 2017.

[11] E. Schrödinger. ¿Qué es la vida?- Edt. Avance. Barcelona, España. 1967.

[12] I. Prigogine. "Tendencias a largo plazo y evolución de la complejidad". En L. Tyrtania, Termodinámica de la supervivencia para las ciencias sociales. Universidad Autónoma Metropolitana. México D.F., 1977.

[13] A. Pérez y C. Moreira. "El papel del desequilibrio en la naturaleza". Foresta Veracruzana. Vol. $4 \mathrm{~N}^{\circ} 2$, pp. 45-51.2002. URL: http://www.redalyc.org/pdf/497/49740207.pdf

[14] C. Marín. "Entropía: un cadaver exquisito". AusArt Journal for Research in Art. Vol. 2, Issue 1, pp. 223-233. June, 2014. URL: http://www.ehu.eus/ojs/index.php/ausart/ article/ view/ 11953 / 11714

[15] R. Sepulveda, P. De la Puente, M. Torres, C. Arditi y P. Muñoz. "Enfoque sistémico y lugar. Una perspectiva para el estudio de hábitat residenciales urbanos". Documento de trabajo FONDECYT 1114-92, Instituto de la Vivienda, Facultad de Arquitectura y Urbanismo, Universidad de Chile, Santiago de Chile. 1992.

[16] S. Rueda. "Modelos e indicadores para ciudades más sostenibles". Fundació Fòrum Ambiental. Cataluña, España. 1999.
[17] S. Boisier. "¿Y si el desarrollo fuese una emergencia sistémica?". Reforma y Democracia $\mathrm{N}^{\circ}$ 27. Octubre 2003. URL: http://www.yorku.ca/ishd/CUBA.LIBRO.06/ DEL/CAPITULO3.pdf

[18] D. Guillén. "Hacia una planificación urbana sistémica. Una experiencia universitaria de aprendizaje y aplicación de nuevos instrumentos técnicos en la planificación urbana tradicional". Espacio y desarrollo. Vol. $1 \mathrm{~N}^{\circ} 20$, pp. 69-86. 2008.

[19] S. Leleur. "Systemic planning. Principles and methology for planning in a complex world”. Schultz Grafisk. Denmark. 2008.

[20] W. Alfonso y L. Galindo. "Evolución de la visión sistémica en el pensamiento urbano del siglo XX La integración de las disciplinas hacia la ciudad sustentable". Facultad de Ciencia Política y Gobierno, Universidad del Rosario, Bogotá. 2011.

[21] E. Solano. "Crítica sistémica. Un enfoque hermenéutico del fenómeno arquitectónico". Revista de Arquitectura. Vol. $16 \mathrm{~N}^{\mathrm{o}} 1$, pp. 68-76. 2014.

[22] L. Ruíz. "Sistemas urbanos complejos acción y comunicación". Cuadernos de investigación urbanística. Vol. 1 No 32, pp. 1-78. 2001. URL: http://polired.upm.es/index.php/ciur/ article/ view/246/242

[23] S. Boccolini. "El evento urbano. La ciudad como un sistema complejo lejos del equilibrio". Quid 16. Vol 1 No 6, pp. 220-252. 2016. URL: http://publicaciones.sociales.uba. ar/index.php/quid16/article/view/2073/1765

[24] J. Fariña y J. Ruíz. "Orden, desorden y entropía en la construcción de la ciudad". Urban. Vol. $1 \mathrm{~N}^{\mathrm{o}}$ 7, pp. 8-15. 2002. URL: http://polired.upm.es/index.php/urban/article/ view/339/339

[25] M. González. "Entropy and negentropy of the particular electric vehicle in urban systems: homeostasis of mobility in Mexico". DYNA. Vol. $85 \mathrm{~N}^{\circ}$ 206, pp. 171-177. 2018.

[26] D. Rapport and A. Friend. "Towards a comprehensive framework for environmental statistics: a stress-responseapproach". Projet d'établissement d'un système général d'information sur l'environment au Canada: l'approche agression-réaction. Ottawa, Canadá: Minister of Supply and Services Canada. 1979. 
[27] A. García, J. Ferman, M. Arredondo, L. Galindo y G. Seingier. "Modelo de planeación ambiental de la zona costera a partir de indicadores ambientales". Sapiens. Revista Universitaria de Investigación. Vol. $6 \mathrm{~N}^{\circ} 2$, pp. 09-23. 2005.

[28] R. Vázquez y R. García. "Indicadores PER y FPEIR para el análisis de la sustentabilidad en el municipio de Cihuatlán, Jalisco, México". Revista de Ciencias Sociales y Humanidades. Vol. 27 N 53-1, pp. 1-26. 2018. URL: http:// dx.doi.org/10.20983/noesis.2018.3.1

[29] OECD. "Core set of indicators for environmental performance reviews. A synthesis report by the Group on the State of the Environment". Environment monographs, $\mathrm{N}^{\circ}$ 83. OCDE/GD (93) 179. 2003. URL. https://www.oecd.org/env/indicatorsmodelling-outlooks/24993546.pdf

[30] OCDE. "Rumo a um Desenvolvimento Sustentável Indicadores Ambientais". Série Cadernos de Referência Ambiental, Centro De Recursos Ambientais, Salvador, Brasil. 2002.

[31] B. Cannon. "Organization for physiological homeostasis". Physiological Reviews. Vol. 9, Issue 3, pp. 399-431. July, 1929.

[32] S. Mahulikar and H. Herwig. "Exact thermodynamic principles for dynamic order existence and evolution in chaos". Chaos, Solitons \& Fractals. Vol. 41, Issue 4, pp. 19391948. 2009. URL: https://www.researchgate. net/publication/222176834_Exact_ thermodynamic_principles_for_dynamic_ order_existence_and_evolution_in_chaos

[33] M. González. "Uber y movilidad urbana en la geografía metropolitana de Guadalajara: Auge y declive". Geograficando. Vol. 13 $\mathrm{N}^{\mathrm{o}}$ 1, pp. 1-14. 2017. URL: https://doi. org/10.24215/2346898Xe020

[34] M. González. "Uberficación y movilidad urbana en el Área Metropolitana de Guadalajara: entropía en las nuevas configuraciones de acceso al transporte motorizada". Ciencia ergo-sum. Vol. $25 \mathrm{~N}^{\circ} 2$, pp. 1-12. 2018. URL: http://cienciaergosum. uaemex.mx/article/view/9500

[35] M. Castells. "La era de la información. Vol. I: la sociedad red". Siglo XXI Editores. México, 2002.

[36] R. Botsman and R. Rogers. "What's Mine is Yours: The Rise of Collaborative
Consumption". Harper Business. New York, E.E.U.U. 2015.

[37] J. Rifkin. "The Zero Marginal Cost Society: the Internet of Things, the Collaborative Commons and the Eclipse of Capitalism". Palgrave Macmillan. Hampshire, United Kindom. 2014.

[38] PBOT (Portland Bureau of Transportation). "A brief history of car sharing". October, 2011. URL: https://www.portlandoregon.gov/ transportation/article/370287? archive $=$ yes

[39] The Guardian. "Story of cities \#30: how this Amsterdam inventor gave bike-sharing to the world". 2016. URL: https://www. theguardian.com/cities/2016/apr/26/storycitiesamsterdam-bi ke-share-scheme

[40] American Public Transportation Association. "Shared mobility and the transformation of public transit". Report was prepared as part of TCRP Project J-11, Task 21, Transit Cooperative Research Program. 2016.

[41] M. Pazos. "El estudio de la movilidad diaria en España, limitaciones en las fuentes y alternativas propuestas". Revista Ería. Vol. 1 $\mathrm{N}^{\circ}$ 66, p. 85-92. 2005.

[42] A. Cohen and S. Shaheen. "Planning for shared mobility". Reports are produced in the Research Department of American Planning Association. 2016.

[43] I. Hartman, D. Keren, A. Abu Dbai, E. Cohen, I. Knapen, A. Yasar and D. Janssens, d. "Theory and Practice in Large Carpooling Problems". 5th International Conference on Ambient Systems, Networks and Technologies (ANT-2014). Procedia Computer Science. Vol. 32, Issue 1, p. 339-347. 2014. DOI: 10.1016/j.procs.2014.05.433.

[44] I. Ballús, S. Shaheen, K. Clonts and D. Weinzimmer. "Peer-to-Peer Carsharing Exploring Public Perception and Market Characteristics in the San Francisco Bay Area, California". Transportation Research Record Journal of the Transportation Research Board. Vol. 1, Issue 2416. pp. 27-36. 2014. DOI: $10.3141 / 2416-04$.

[45] K. Degirmenci and M. Breitner. "Carsharing: A literature review and a perspective for information systems research". In Beckmann, Lars (Ed.) Multikonferenz Wirtschaftsinformatik (MKWI 2014), pp. 26-28. Paderborn, Germany. February, 2014. 
[46] P. Dimaggio and W. Powell. "The iron cage revisited: institutional isomorphism and collective rationality in organizational fields". American Sociological Review. Vol. 48, Issue 2, pp. 147-160. 1983. DOI: 10.2307/2095101.

[47] R. Llamas. "Un análisis institucional de la implantación de la agenda local 21 por los ayuntamientos españoles". Tesis de doctorado. Editorial de la Universidad de Granada, Granada, España. 2005.

[48] M. Suchman. "Managing legitimacy: strategic and institutional approaches".
Academy of Management Review. Vol. 20, Issue 3, pp. 571-610. 1995. DOI: $10.2307 / 258788$.

[49] P. Landoni. "Isomorfismo y calidad: redefiniendo los espacios públicos y privados en la educación superior uruguaya". Revista Uruguaya de Ciencia Política. Vol. $17 \mathrm{~N}^{\circ} 1$, pp. 183-202. 2008.

[50] B. Herrera. "La calidad de las instituciones de educación superior (IES) privadas en zacatecas: relevancia y eficiencia". Tesis de doctorado. Universidad Nacional Autónoma de México, México. 2010. 